\title{
Radiological Study of Posterior Cerebral Artery Variations and its Influence on Cerebral Stroke
}

\section{Dravya Jayakumar ${ }^{1}$, Radhika P.M. ${ }^{2}$, Shailaja Shetty ${ }^{3}$}

\section{ABSTRACT}

Background: The posterior cerebral artery (PCA) is the terminal branch of the basilar artery. The fetal variant of PCA (fPCA) arises directly from the internal carotid artery. A fPCA can be either complete or partial with a hypoplastic segment connecting to the basilar artery. Individuals with fPCA could be more prone to ischemic strokes.

Aims: This study aims to analyze the posterior cerebral artery variations on Magnetic resonance (MR) angiogram and its influence on ischemic strokes.

Materials and Mmethods: The MR angiogram images of 150 patients - 75 with evidence stroke and 75 without evidence of stroke were analyzed for the presence of fPCA. The origin, diameter, and variations of PCA and posterior communicating artery were analysed for evidence of the presence of $\mathrm{PPCA}$.

Results: There were 20 individuals with fPCA out of 75 individuals with evidence of stroke and 9 individuals with fPCA among 75 individuals without evidence of stroke. The presence of fPCA associated with stroke is statistically significant with P-value of 0.0375 .

Conclusions: The presence of fPCA in an individual is one of the predisposing factors to stroke.

Key Words: Fetal posterior cerebral artery, Angiography, Stroke

\section{INTRODUCTION}

The cerebral circulation is mainly by the internal carotid artery(ICA) and the vertebro-basilar system which unite to form the circle of Willis(CoW). The posterior cerebral artery (PCA) is the terminal branch of the basilar artery that supplies the posteromedial surface of the temporal lobe and the occipital lobe. Posterior communicating artery (PCoM) anastomoses the PCA and ICA and forms the posterior part of $\mathrm{CoW}^{1}$. The PCA has four segments and six cortical branch$\mathrm{es}^{2}$. The pre-communicating segment (P1) of PCA extends from its origin to PCoM. The fetal variant of PCA(fPCA) arises directly from the ICA ${ }^{3}$.

A fPCA can be either complete; or partial with a connection to the basilar artery through a hypoplastic P1 segment. These variants can be unilateral or bilateral ${ }^{4}$. The partial type of fPCA can be sub-divided based on the diameter of contributing arteries into embryonic, adult, and transitional configurations. When the PCA diameter is less than PCoM it is said to embryonic configuration. If the PCA diameter is equal to $\mathrm{PCoM}$ it is said to be transitional configuration. Whereas if the diameter of PCA and PCoM are equal it is called adult ${ }^{5}$. The fPCA could be a developmental anomaly as in cases of complete PPCA or acquired due to vascular conditions like atherosclerotic diseases in cases of partial fPCA. It could also be accompanied by other CoW variations. The variation in blood vessels alters the flow dynamics and development of collateral vessels ${ }^{6,7}$.

In individuals with fetal-type PCA, the lack of proper collaterals may hemodynamically compromise the anterior as well as posterior cerebral circulation. In cases of complete PPCA the entire posterior circulation depends on the ICA which can lead to hypoperfusion. This would mean that the incidence of strokes could be more in such a population than the population with normal cerebral circulation. Magnetic resonance angiography (MRA) of the brain can provide information on the anatomy of cerebral circulation ${ }^{2}$. The aim of the study is to evaluate if individuals with partial and complete

\section{Corresponding Author:}

Dr. Radhika P.M., Assistant professor,Department of Anatomy, M.S.Ramaiah Medical College, MSRIT Post Bangalore, 560054, India. Phone: 9035044023; E-mail: parameshradhika@gmail.com

ISSN: $2231-2196$ (Print)

ISSN: 0975-5241 (Online)

Received: 16.05 .2020

Revised: 13.06 .2020

Accepted: 24.06 .2020

Published: 22.07 .2020 
fetal-type posterior cerebral artery could be more prone to ischemic strokes on Magnetic resonance (MR) angiogram.

\section{MATERIALS AND METHODS}

A case-control study was conducted on patients who underwent MRA at Ramaiah Hospitals from 2014 to 2018. Patients who underwent MRA with evidence of stroke were grouped in one category and those without evidence of stroke were grouped in the second category. A total of $150 \mathrm{MR}$ angiograms were analyzed - 75 with evidence of stroke and 75 without evidence of stroke. The images were analyzed for evidence of full or partial fPCA and ischemic strokes. Patients with a history of head trauma, previous illness, cerebral surgery, arteriovenous malformations, and vasculitis syndromes were excluded from this study.

The origin, diameter, course, and variations of PCA were analyzed for evidence of the presence of PPCA in the two subgroups - with evidence of stroke and without evidence of stroke. Ischemic strokes or infarction on MR scan images were defined as areas of low density in the arterial territory.

Statistical analysis of data -SPSS software was used for statistical analysis. Descriptive statistics of fPCA was analyzed and summarized in terms of percentage. Fisher's exact test was used to find an association between the presence of fPCA and stroke. The Odds ratio was calculated.

\section{RESULTS}

150 MR angiograms were studied which included 75 with evidence of stroke and 75 without evidence of stroke. There were 60 females and 90 males with a mean age of 58.14 years.

There were 20 individuals with fPCA out of 75 individuals with evidence of stroke. There were 6 complete PPCAs and 14 partial fPCAs. Out of the 20 individuals, 3 had bilateral fPCA and 17 had unilateral fPCA - 9 right-sided and 8 leftsided. In individuals without evidence of stroke, there were 9 individuals with fPCA out of 75 individuals. There were 3 complete fPCAs and 6 partial fPCAs. Out of the 9 individuals, 2 had bilateral fPCA and 7 had unilateral fPCA - 5 rightsided and 2 left-sided. (Table 1)

The presence of PPCA associated with stroke is statistically significant with P-value of 0.0375 . The odds ratio was calculated to be 2.66 .

\section{DISCUSSION}

The Circle of Wills (CoW) is the anastomosis of vessels supplying the cerebral area (fig 1). The PCA anastomoses with
PCoM to form the posterior part of CoW. It creates an alternate blood supply from both ICA and basilar artery to the posterior circulation. Variations in the CoW cause a compromise in blood flow during hemodynamically unstable states. A study conducted by Naveen SR et al. showed that only $16.6 \%$ of the study population had a complete CoW on $M^{2} A^{8}$. In our study, we have focused on the fPCA variant only.

The fPCA arises directly from the ICA. It can either be complete or partial; unilateral or bilateral (fig 2,3). It is a fairly common variant in the population. A study conducted by Chauhan et al. on MR angiograms found the incidence of fPCA to be $54.3 \%{ }^{3}$. Another study by Lochner et al. reviewed the prevalence of fPCA to be between $15 \%$ and $46 \%$ based on both anatomic and angiographic studies 9 . In our study, the total incidence of fPCA was found to be $19.33 \%$.

The presence of fPCA could predispose the individual to stroke by various factors. Some of the proposed factors for this association are altered flow dynamics, lack of development of leptomeningeal collaterals ${ }^{10,11}$. A study conducted by Wentland et al. showed that individuals with PPCA had asymmetrically smaller perfusion transit parameters in the brain $^{12}$. A study by de Monyé et al. also hypothesised that patients with stroke in posterior circulation have a PPCA in combination with high-grade stenosis of the ipsilateral ICA $^{13}$. However, they did not find any statistically significant association between them.

A study conducted by Arjal et al. on CT angiograms of the brain found the incidence of PPCA to be $36.2 \%$ in individuals with stroke and $22.7 \%$ in individuals without stroke. The odds ratio for stroke was found to be 3.027 with partial fPCA and 1.448 with complete PPCA in their study ${ }^{6}$. In our study, on MR angiograms of the brain, the incidence of fPCA was $26.6 \%$ in individuals with stroke and $12 \%$ in individuals without evidence of stroke. Our results show that individuals with fPCA have 2.66 times the odds of stroke (Table 2).

The posterior circulation in cases of complete fPCA is dependent completely on the ICA. This could mean more chances of hypoperfusion in cases of complete fPCA than in those with partial fPCA. However, in our study, the incidence of partial fPCA with stroke was found to be higher than that of complete fPCA.

We also found that unilateral fPCA was more frequent than bilateral fPCA. These findings are consistent with studies conducted by Chauhan et al., Arjal et al. ${ }^{4,5}$. The incidence of right-sided fPCA (12.6\%) was higher than left-sided PPCA $(10 \%)$. These findings are consistent with studies done by Chauhan et al., Amir et al., ${ }^{4,6}$.

The fPCA predisposes the individual to stroke and this association can help monitor individuals with this variant closely and provide appropriate measures for prevention and surgi- 
cal management of stroke. Recognition of fPCA is important to prevent errors in cerebral perfusion studies interpretation, enhance pre-operative planning in the surgical approach to minimize the risk of perioperative cerebral hypoperfusion or embolization. The clinical influence of the presence of PPCA on the outcome of ischemic stroke patients could be further studied.

The major limitation of this study is that it cannot determine whether the altered cerebral blood flow was the only cause for stroke in these individuals. The other limitations include a small sample size and the possibility of some selection bias.

\section{CONCLUSION}

The presence of fPCA and its association with stroke was found to be statistically significant in our study. Individuals with fPCA have higher odds of developing stroke. Recognizing this variant is important for coming up with appropriate preventive measures and management of stroke. It is also important for surgeons to recognize this variant to minimize complications.

Acknowledgement: Authors acknowledge the immense help received from the scholars whose articles are cited and included in references of this manuscript. The authors are also grateful to authors / editors / publishers of all those articles, journals and books from where the literature for this article has been reviewed and discussed.

\section{Source of Funding: Nil}

Conflict of interest : No conflict of interest

\section{REFERENCES}

1. Pai BS, Varma RG, Kulkarni RN, Nirmala S, Manjunath LC, Rakshith S. Microsurgical anatomy of the posterior circulation. Neurol India. 2007;55(1):31-41.
2. Cilliers K, Page B. Variation and Anomalies of the Posterior Cerebral Artery: Review and Pilot Study. Turk Neurosurg.2017 Dec 14.

3. Chauhan K, Khare S, Jain S, Sharma A, Yadav A. A study of partial fetal posterior cerebral artery through cerebral MRA-incidence and clinical significance. J Anat Sciences. 2015; 23(2): 6-10.

4. Nouh A, Remke J, Ruland S. Ischemic Posterior Circulation Stroke: A Review of Anatomy, Clinical Presentations, Diagnosis, and Current Management. Frontiers in Neurology. 2014;5:30.

5. Saeki N, Rhoton AL Jr: Microsurgical Anatomy Of The Upper Basilar Artery And The Posterior Circle Of Willis. J Neurosurg, 1977; 46: 563-578.

6. Arjal RK, Zhu T, Zhou Y.The study of fetal-type posterior cerebral circulation on multislice CT angiography and its influence on cerebral ischemic strokes.Clin Imaging. 2014; 38(3):221-5.

7. Ardakani SK, Dadmehr M, Nejat F, et al. The cerebral arterial circle (circulus arteriosus cerebri): an anatomical study in fetus and infant samples. Pediatr Neurosurg. 2008;44(5):388-392.

8. Naveen SR, Bhat V, Karthik GA. Magnetic resonance angiographic evaluation of circle of Willis: A morphologic study in a tertiary hospital set up. Ann Indian Acad Neurol. 2015;18(4):391-397.

9. Lochner P, Golaszewski S. Caleri, F, et al. Posterior circulation ischemia in patients with fetal-type circle of Willis and hypoplastic vertebrobasilar system. Neurol Sci 2011;32(6):1143-6.

10. van Raamt AF, Mali WP, van Laar PJ, van der Graaf Y. The fetal variant of the circle of Willis and its influence on the cerebral collateral circulation. Cerebrovasc Dis. 2006;22(4):217-24.

11. Lambert SL, Williams FJ, Oganisyan ZZ, Branch LA, Mader EC. Fetal-Type Variants of the Posterior Cerebral Artery and Concurrent Infarction in the Major Arterial Territories of the Cerebral Hemisphere. J Investig Med High Impact Case Rep. 2016 Jul-Sep; 4(3).

12. Wentland A, Rowley H, Vigen K, Field A. Fetal origin of the posterior cerebral artery produces left-right asymmetry on perfusion imaging. Am J Neuroradiol.2010; 31:448-453.

13. de Monyé C, Dippel DWJ, Siepman TAM et al. Is a fetal origin of the posterior cerebral artery a risk factor for TIA or ischemic stroke? J Neurol 255, 239-245 (2008).

Table 1: Percentage of PCA and PPCA in patients with stoke and without stoke

\begin{tabular}{|c|c|c|c|}
\hline Category & PCA & fPCA & \\
\hline With evidence of stroke & $55(73.3 \%)$ & $20(26.6 \%)$ & \\
\hline & & Unilateral -17 & Bilateral - 3 \\
\hline & & Complete - 6 & Partial - 14 \\
\hline Without evidence of stroke & $66(8$ & $9(12 \%)$ & \\
\hline & $8 \%)$ & Unilateral - 7 & Bilateral - 2 \\
\hline & & Complete - 3 & Partial - 6 \\
\hline
\end{tabular}

P value-0.037

Odds ratio-2.66. 
Table 2: Comparison of data with previous studies

\begin{tabular}{lllcl} 
Study & Sample size & fPCA & P value & Odds ratio \\
Chauhan et al. ${ }^{3}$ & 92 MR angiograms & $54.3 \%$ & - & - \\
Arjal et al. ${ }^{4}$ & 203 CT angiograms & $\mathrm{S}-36.2 \%$ & 0.0307 & partial fPCA- 3.027 \\
& $(58 \mathrm{~S}+145 \mathrm{NS})$ & $\mathrm{NS}-22.7 \%$ & 0.391 & complete fPCA - 1.448 \\
& & & 0.0552 & Both - 1.926 \\
Present study & $150 \mathrm{MR}$ angiograms & $\mathrm{S}-26.6 \%$ & 0.0375 & 2.66 \\
& $(75 \mathrm{~S}+75 \mathrm{NS})$ & $\mathrm{NS}-12 \%$ & & \\
\hline
\end{tabular}

S - Stroke, NS - Non Stroke

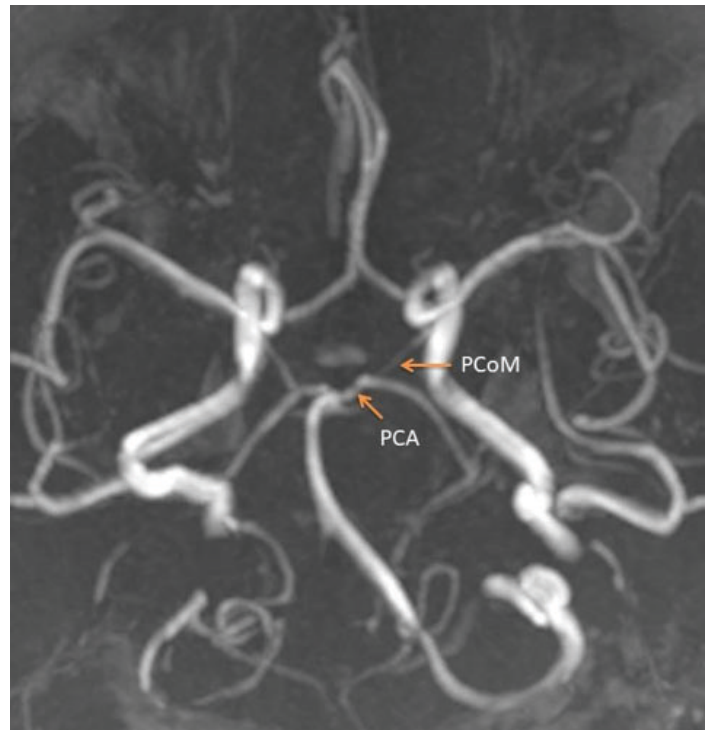

Figure 1: MR angiogram of Normal circle of Willis (PCA arises from basilar artery and is greater than PCoM).

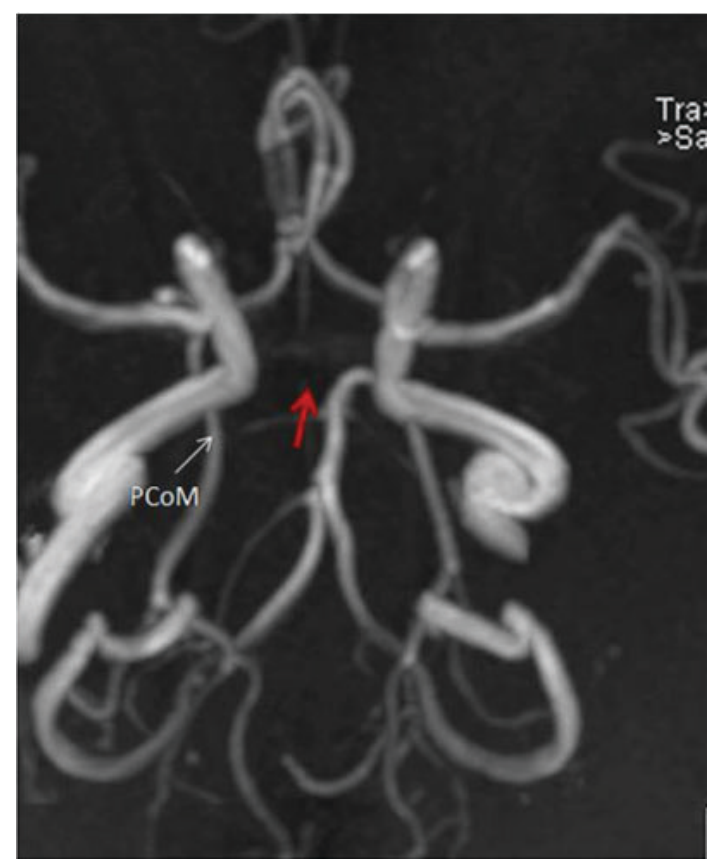

Figure 2: MR angiogram Complete Right fPCA. Red arrow shows the absence of pre-communicating segment of the PCA. PCoM is continuing as PCA.

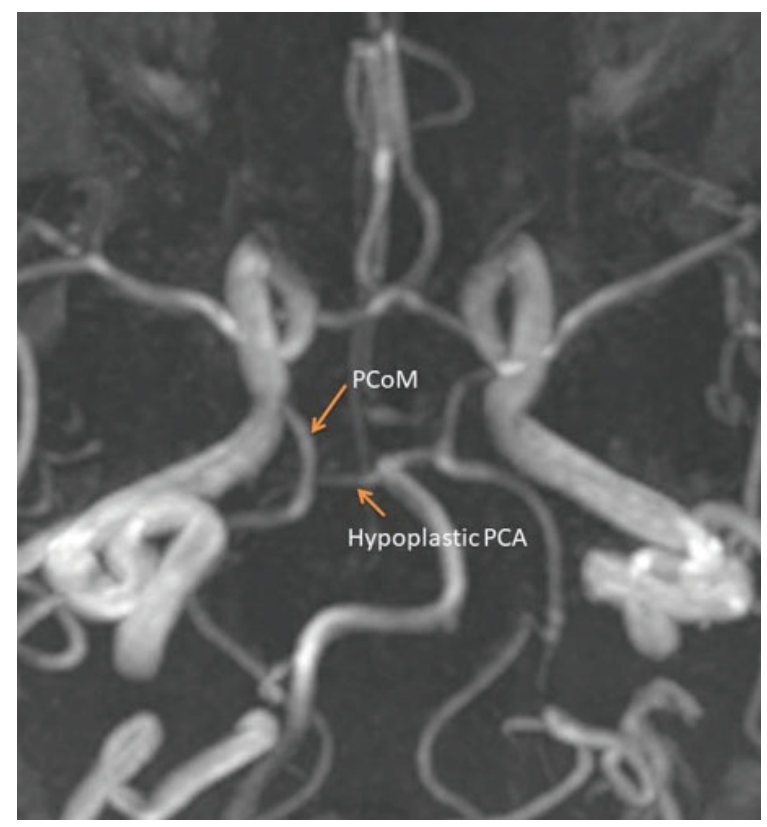

Figure 3: MR angiogram partial Right fPCA. Hypoplastic $P C A$ is seen. 